\title{
Development of a dense SNP-based linkage map of an apple rootstock progeny using the Malus Infinium whole genome genotyping array
}

Laima Antanaviciute ${ }^{1}$, Felicidad Fernández-Fernández ${ }^{1}$, Johannes Jansen², Elisa Banchi ${ }^{3}$, Katherine M Evans ${ }^{4}$, Roberto Viola ${ }^{3}$, Riccardo Velasco ${ }^{3}, \operatorname{Jim}_{\mathrm{M}}$ Dunwell ${ }^{5}$, Michela Troggio $^{3}$ and Daniel J Sargent ${ }^{3^{*}}$

\begin{abstract}
Background: A whole-genome genotyping array has previously been developed for Malus using SNP data from 28 Malus genotypes. This array offers the prospect of high throughput genotyping and linkage map development for any given Malus progeny. To test the applicability of the array for mapping in diverse Malus genotypes, we applied the array to the construction of a SNP-based linkage map of an apple rootstock progeny.

Results: Of the 7,867 Malus SNP markers on the array, 1,823 (23.2\%) were heterozygous in one of the two parents of the progeny, 1,007 (12.8\%) were heterozygous in both parental genotypes, whilst just 2.8\% of the 921 Pyrus SNPs were heterozygous. A linkage map spanning 1,282.2 cM was produced comprising 2,272 SNP markers, 306 SSR markers and the S-locus. The length of the M432 linkage map was increased by $52.7 \mathrm{cM}$ with the addition of the SNP markers, whilst marker density increased from $3.8 \mathrm{cM} /$ marker to $0.5 \mathrm{cM} /$ marker. Just three regions in excess of $10 \mathrm{cM}$ remain where no markers were mapped. We compared the positions of the mapped SNP markers on the M432 map with their predicted positions on the 'Golden Delicious' genome sequence. A total of 311 markers (13.7\% of all mapped markers) mapped to positions that conflicted with their predicted positions on the 'Golden Delicious' pseudo-chromosomes, indicating the presence of paralogous genomic regions or mis-assignments of genome sequence contigs during the assembly and anchoring of the genome sequence.

Conclusions: We incorporated data for the 2,272 SNP markers onto the map of the M432 progeny and have presented the most complete and saturated map of the full 17 linkage groups of M. pumila to date. The data were generated rapidly in a high-throughput semi-automated pipeline, permitting significant savings in time and cost over linkage map construction using microsatellites. The application of the array will permit linkage maps to be developed for QTL analyses in a cost-effective manner, and the identification of SNPs that have been assigned erroneous positions on the 'Golden Delicious' reference sequence will assist in the continued improvement of the genome sequence assembly for that variety.
\end{abstract}

Keywords: Infinium, Golden Gate, Breeding, Selection, Genome sequence, Marker

\section{Background}

The cultivated apple, Malus pumila Mill. $(2 n=2 x=34)$, is a member of the Spireaoideae subfamily of Rosaceae, and is the fourth most economically-important fruit crop worldwide. As a consequence of the long juvenility phase of apple trees and of relatively high husbandry

\footnotetext{
* Correspondence: dan.sargent@iasma.it

${ }^{3}$ Istituto Agrario San Michele all'Adige, Via E. Mach 1, 38010 San Michele all'Adige, Italy

Full list of author information is available at the end of the article
}

costs, the breeding and selection of novel apple rootstocks and scions is a time-consuming and costly procedure. Marker assisted selection (MAS) has the potential to increase the precision of apple breeding and an essential prerequisite to MAS is the production of high quality saturated genetic linkage maps to enable marker-trait associations to be made.

The genome of the apple scion cultivar 'Golden Delicious' was recently sequenced and assembled into 17 pseudo-chromosomes by an international consortium [1]

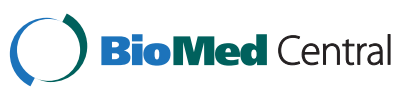


using predominantly second generation 454 sequencing technology. Despite the inherent difficulties associated with contig assembly and gene-prediction in complex heterozygous genomes [2], the 'Golden Delicious' genome covers $598.3 \mathrm{Mbp}$, an estimated $71.2 \%$ of the Malus genome and almost complete coverage of the gene-space of the variety [1], with an average depth of sequencing $16.9 \times$. Thus, the sequence provides a solid foundation for a wealth of downstream research activities including marker development, linkage map construction and marker-trait association.

The availability of data relating to single nucleotide polymorphisms (SNPs) from early in the development of the 'Golden Delicious' genome sequence meant that assays have been developed for screening segregating SNPs in apple mapping populations at relatively high throughput using the SNPlex and Golden Gate genotyping platforms $[3,4]$, permitting not only the rapid development of linkage maps for Malus progenies, but the high resolution anchoring orientation of the scaffolded sequence data to a reference linkage map [1]. The degree of transferability of heterozygous SNPs between Malus varieties and species, and between Malus and Pyrus has recently been assessed [5], and estimates have been made about the number of SNPs required to allow the construction of a saturated linkage map in any given Malus progeny. In the study of Micheletti et al. [5], SNPs identified from the 'Golden Delicious' genome sequence were validated and tested for heterozygous transferability $\left(\mathrm{T}_{\mathrm{SNP}}\right)$ in a diverse selection of Malus germplasm. The investigation showed that SNPs identified within the 'Golden Delicious' sequence had an average transferability rate of $40.9 \%$ to Malus cultivars, with the lowest $\mathrm{T}_{\mathrm{SNP}}$ to the cultivar 'Wagner' (25.7\%) from those tested. The transferability rate in rootstock germplasm was between $29.9 \%$ and $39.7 \%$, whilst to an accession of Pyrus pyrifolia, the $\mathrm{T}_{\mathrm{SNP}}$ value was just $1.8 \%$, demonstrating low cross-genera SNP transferability of the SNPs tested [5].

Through international collaboration, led by the RosBREED initiative in the USA [6], the Malus research community has developed an Infinium ${ }^{\circledR}$ II WGG genotyping array (referred to hereafter as the International RosBREED SNP Consortium array; IRSC array) for Malus and Pyrus using data from the re-sequencing of 27 Malus genotypes along with data from the 'Golden Delicious' genome sequence. The IRSC array contains a total of 7,867 Malus SNPs [7] in addition to 921 Pyrus SNPs. The development of this array represents a milestone in the development of molecular genetics and genomics resources for Malus and offers the promise of rapid, low-cost, high-throughput genotyping for the purposes of linkage map construction and the genotyping of germplasm collections, that will facilitate future QTL and genome-wide association studies. However, reports are yet to emerge of the efficacy of such arrays for genotyping germplasm and mapping populations from divergent sources or from species related to those for which they were originally designed.

The M432 mapping progeny [8,9] has been raised for the study of genes controlling traits of relevance to rootstock breeding, with the long-term aim of developing robust markers for MAS. Since the progeny is derived from parental rootstock varieties, the genetic basis of the seedlings that comprise the population represents a departure from the well-characterised scion genotypes that were used to identify SNPs for the construction of the IRSC array [7]. The progeny has been previously characterised with $S$-locus-specific markers, and 323 (306 co-dominant, and 17 dominant) SSR markers [9] distributed throughout the Malus genome. A comprehensive consensus linkage map of the progeny has been developed spanning 17 linkage groups (LGs), with an average marker density of one marker every $3.79 \mathrm{cM}$. The linkage map was partially anchored to the 'Golden Delicious' genome sequence, and a total of $47 \%$ of the sequence that was contained in metacontigs could be assigned positions on the M432 map.

The aim of this investigation was to test the IRSC array in the M432 rootstock mapping progeny to determine its utility to genome-wide saturated map construction. Additionally, we aimed to increase the percentage of the Malus genome sequence that could be directly related to regions of the M432 linkage map for the purposes of candidate gene identification and marker development following QTL analysis. We evaluated the SNP-based linkage map produced against the previously-published SSR-based linkage map of the population developed by FernándezFernández et al. [9]. We compared the positions of SNP markers on the M432 linkage map with their predicted positions on the 'Golden Delicious' genome sequence and assessed the accuracy of the genomic placement of the heterozygous SNP markers in relation to the genetic positions of the markers on the M432 map. An evaluation was made of the ease at which the IRSC array could be implemented in the mapping progeny in relation to previous SSR assays performed for linkage map construction in this progeny.

\section{Results}

SNP heterozygosity in the M432 mapping progeny

GenTrain scores for all SNPs scored in the M432 progeny ranged from 0.043 to 0.961 , with an average of 0.723 . Cluster separation ranged from 0.004 to 1 with an average of 0.814 . Of the 8,788 SNP markers contained on the IRSC array (7,867 Malus SNPs and 921 Pyrus SNPs), 664 markers failed in both parental genotypes, 44 failed in the 'M.27' genotype and a further 51 markers 
failed in the 'M.116' genotype. A total of 5,078 markers were homozygous in both parental genotypes of the M432 progeny, and 95 revealed unexpected genotypes in the progeny given the parental genotypes. Of the remaining 2,856 markers 1,007 were heterozygous in both parents, 976 markers were heterozygous only in the 'M.27' parental genotype, and 873 markers were heterozygous only in 'M.116'.

\section{SNP and SSR co-segregation analysis and linkage map construction}

GenTrain scores for the markers considered for mapping ranged from 0.4 to 0.961 , with an average of 0.713 . Cluster separation scores ranged from 0.347 to 1 , with an average of 0.848. A total of 854 SNPs heterozygous in the 'M.27' parental genotype, 751 SNPs heterozygous in the 'M.116' parental genotype, and a further 665 SNPs heterozygous in both parental genotypes of the M432 progeny coalesced into the 17 expected LGs for a consensus genetic linkage map. Thus, a total of 586 putatively segregating loci were not located to LGs on the M432 map. Close visual inspection of these loci in GenomeStudio (Illumina) revealed that the majority had poor cluster separation scores and did not cluster as expected despite having high GenTrain scores. In many cases, the clusters that were produced were composed of a number of smaller sub-clusters and of individual genotype calls that did not cluster tightly or that did not cluster in the expected region of the graph space and were shifted from either the 0 or the 1 axis (Figure 1). As such, cluster patterns at these loci were either unsuitable for genotypic analysis due to mis-assignation of sub-clusters, or it was not possible to reliably determine the heterozygosity status of the parental genotypes, and thus the loci were not considered for further analyses.

In addition to the 2272 SNPs, the 306 codominant SSR markers and the $S$-locus that were previously mapped by Fernández-Fernández et al. [9] were located to the 17 LGs. Previously mapped dominant SSR markers segregating $A \varnothing \times A \varnothing$ were not considered for mapping in this investigation. The consensus linkage map of the M432 progeny is presented in Figure 2 and Table 1 summarises the marker composition and lengths of the 17 M432 LGs. The map spanned a total of 1,282.2 $\mathrm{cM}$ and contained a total of 2,579 molecular markers. LG 14 was the shortest LG on the map, spanning $56.1 \mathrm{cM}$, whilst LG15 was the longest, spanning $132.4 \mathrm{cM}$. The highest number of markers mapped to a single LG was 221 on LG2; the smallest number of markers on a single LG was 69 on LG4. The map had an average marker density of one marker every $0.5 \mathrm{cM}$. The linkage map contained a total of three regions in excess of $10 \mathrm{cM}$ that contained no mapped molecular markers.

The largest region to which no markers were mapped was $26.6 \mathrm{cM}$ on LG4 which was defined by four SSR markers that had been previously mapped by FernándezFernández et al. [9]. Similarly, a region at the distal end of LG16 spanning $26.5 \mathrm{cM}$ containing no markers was defined by a single SSR marker previously mapped by Fernández-Fernández et al. [9]. Upon closer inspection, the segregation of these SSR loci were distorted towards an excess of an allele from 'M.27' parental genotype which could have potentially led to an overestimation of the genetic distance between these markers and their closest flanking markers along the LG. The markers were tentatively included on the linkage map presented

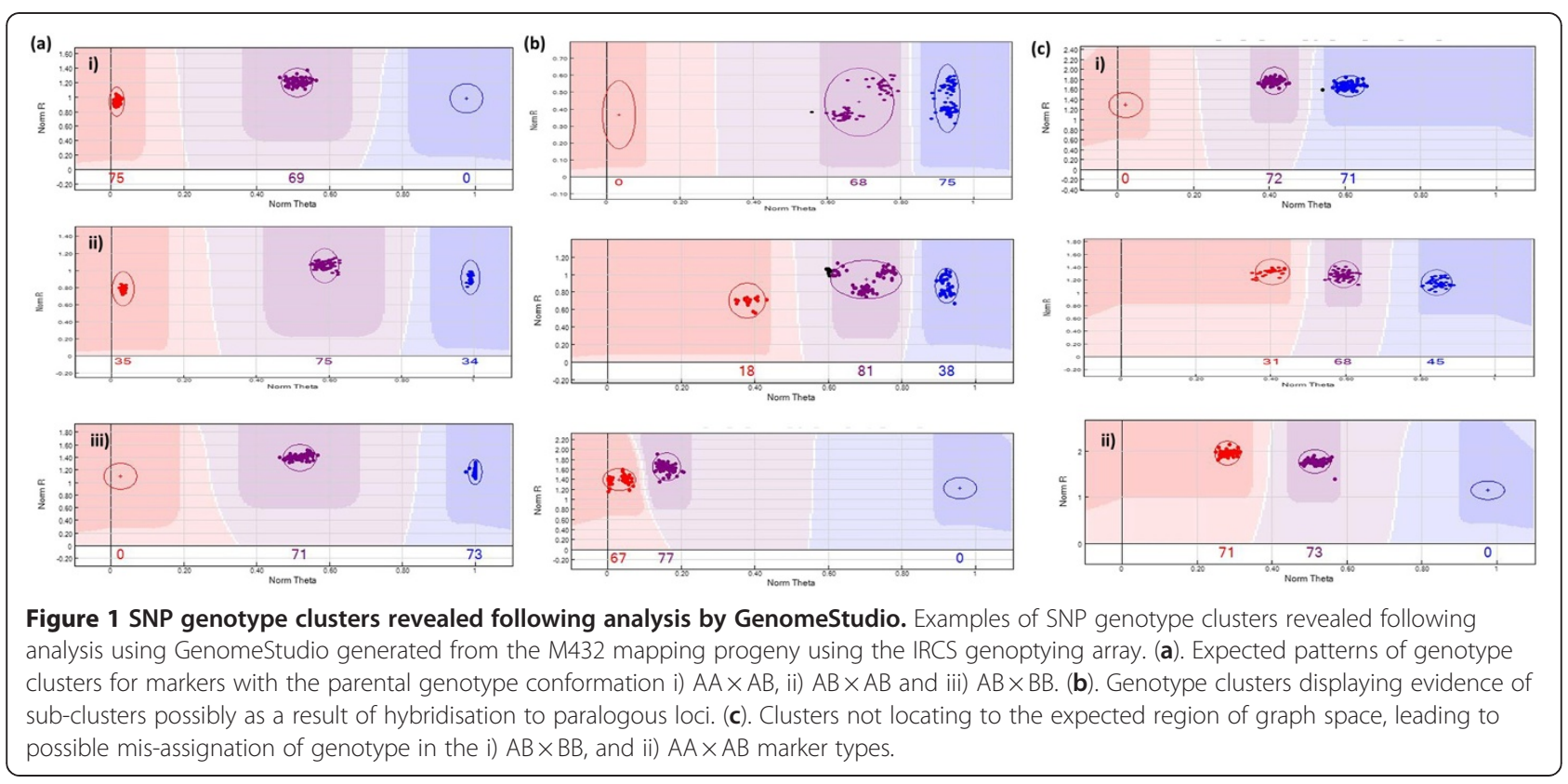




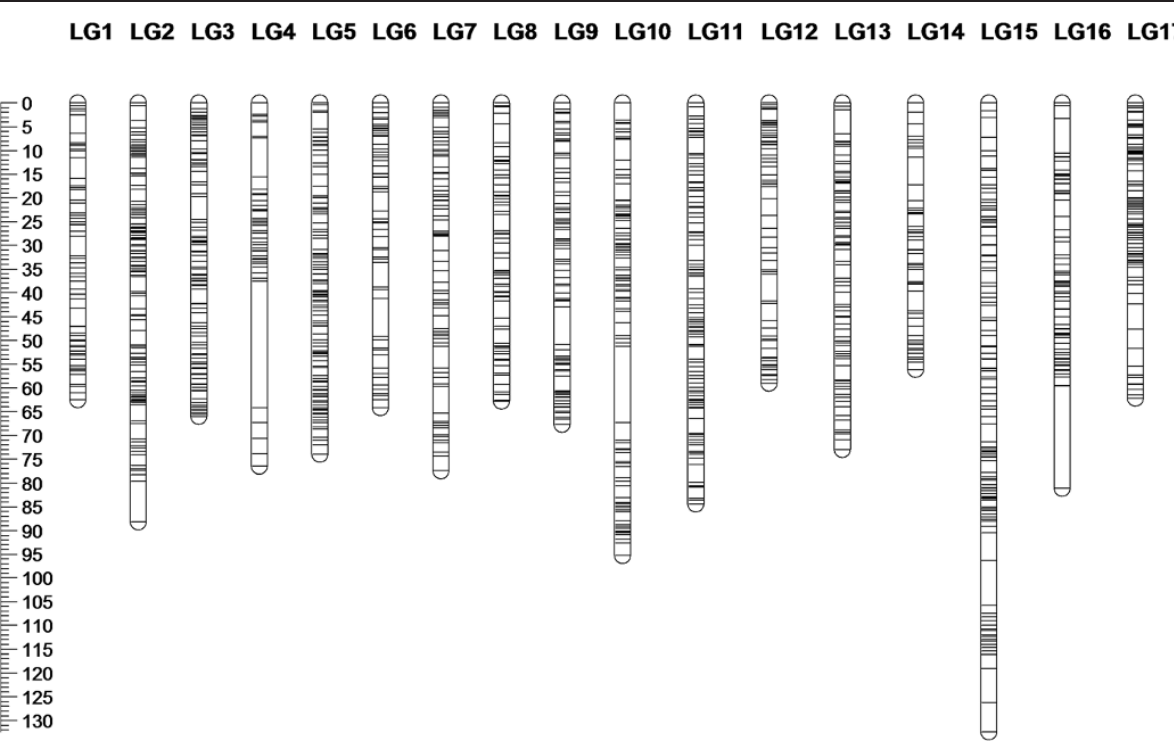

Figure 2 SNP-based linkage map of the M432 progeny. An consensus genetic linkage map of the M432 Malus mapping population composed of 2,579 molecular markers, including 2,272 SNPs generated with the IRSC array, 306 SSRs and the S-locus, spanning 1,282.2 cM over $17 \mathrm{LGs}$. The scale in centi-Morgans is given at the edge of the figure.

since their position and order corresponded with the previously published M432 linkage map.

Figure 3 shows a comparison of the order of SSR markers in the M432 map between the current investigation and the report of Fernández-Fernández et al.
[9]. Marker order of previously mapped SSRs in the M432 progeny was generally well conserved, with only minor changes compared to the previous SSR map of Fernández-Fernández et al. [9] in regions where the SSR markers were tightly linked (generally

Table 1 The number of markers and the genetic distances mapped on the M432 SNP-based linkage map

\begin{tabular}{|c|c|c|c|c|c|c|c|}
\hline Linkage group & Length & ABXAA SNP & AAXAB SNP & $A B \times A B$ SNP & SSR & Total markers & Segregation distortion \\
\hline 1 & 62.459 & 24 & 43 & 42 & 14 & 123 & $0-3 \mathrm{cM} ; 12$ markers \\
\hline 2 & 79.641 & 34 & 98 & 74 & 15 & 221 & \\
\hline 3 & 66.027 & 75 & 53 & 54 & 14 & 196 & 31-76 cM; 13 markers \\
\hline 4 & 76.508 & 16 & 12 & 33 & 8 & 69 & \\
\hline 5 & 81.756 & 73 & 44 & 52 & 23 & 192 & \\
\hline 6 & 64.207 & 41 & 43 & 23 & 12 & 119 & \\
\hline 7 & 77.391 & 40 & 31 & 43 & 15 & 129 & $0-5 \mathrm{cM} ; 6$ markers \\
\hline 8 & 62.792 & 46 & 39 & 22 & 12 & 119 & \\
\hline 9 & 67.72 & 46 & 40 & 47 & 25 & 158 & 7-57 cM; 36 markers \\
\hline 10 & 95.275 & 25 & 41 & 42 & 25 & 133 & \\
\hline 11 & 84.42 & 66 & 63 & 43 & 28 & 200 & \\
\hline 12 & 59.003 & 19 & 22 & 51 & 16 & 108 & $\begin{array}{l}10-16 \mathrm{cM}, 20-36 \mathrm{cM}, 49 \mathrm{cM}, \\
54-58 \mathrm{cM} ; 33 \text { markers total }\end{array}$ \\
\hline 13 & 73.039 & 66 & 44 & 31 & 16 & 157 & \\
\hline 14 & 56.136 & 82 & 18 & 22 & 13 & 135 & \\
\hline 15 & 132.444 & 69 & 65 & 39 & 25 & 198 & \\
\hline 16 & 81.134 & 52 & 66 & 21 & 20 & 159 & \\
\hline 17 & 62.242 & 82 & 29 & 26 & $26^{*}$ & 163 & 31-61 cM; 13 markers \\
\hline Total & 1282.194 & 854 & 751 & 665 & $307^{*}$ & 2579 & \\
\hline
\end{tabular}

The total number of SNP and SSR markers mapped in the M432 mapping progeny, the number of markers per chromosome and the total length of each LG in centi-Morgans (CM). 


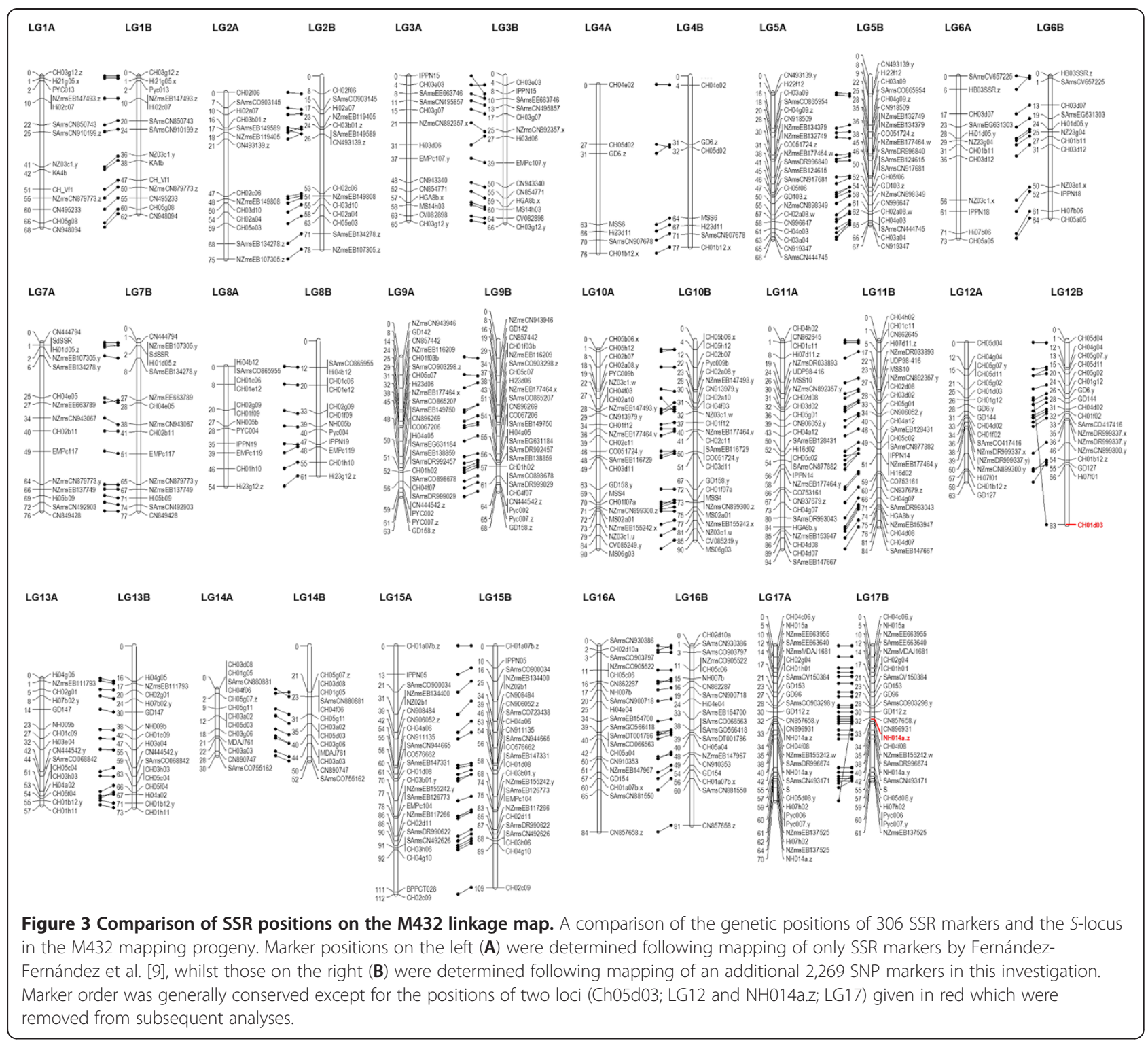

where they mapped less than $2 \mathrm{cM}$ apart). The exceptions to this trend were loci Ch01d03, which was located at $25 \mathrm{cM}$ on LG12 on the map presented by Fernández-Fernández et al. [9], but which was located $27.3 \mathrm{cM}$ away from its closest marker at the distal end of LG12 in this investigation, and NH014a.z, which was located at $33.3 \mathrm{cM}$ in this investigation on LG17, but was located at the distal end of LG17 on the map of Fernández-Fernández et al. [9], $6 \mathrm{cM}$ from its closest flanking marker. The positions of these markers are presented in red on Figure 3 for comparison, but the markers were removed from the final analysis of the consensus SNP and SSR map presented in Figure 2. The addition of SNP markers to the consensus map reduced the effective comparable length of the M432 SSR map by a total of
$74.5 \mathrm{cM}(6.1 \%)$, but due to the increased coverage of the genome with SNP markers at the ends of LGs where no SSRs had previously been mapped, overall, the linkage map length was increased by $52.7 \mathrm{cM}$ (4.3\%). Additional file 1 details the segregating markers mapped in the M432 population including the dbSNP (EMBL) accession code assigned to each on the IRSC array, the LG and map position of each mapped marker, the monogenic marker segregation ratios, associated chi-squared values and predicted pseudo-chromosome positions of each marker on the 'Golden Delicious' genome sequence.

\section{Segregation distortion on the M432 linkage map}

A total of six LGs exhibited some level of segregation distortion along their length (Table 1 and Additional file 1). 
Of these, two (LG1 and LG7) displayed segregation distortion for a short region at their proximal end, whilst the distortion observed on the other four groups (LG3, LG9, LG12 and LG17) was more extensive, the most significant distortion present on LG12 and on LG17 in the region of the $S$-locus.

\section{Comparison of genetic and physical positions of the IRSC array SNP markers}

Figure 4 depicts the physical distances of the mapped SNP markers on the 'Golden Delicious' genome sequence plotted against their genetic positions on the M432 map. In general, the within LG genetic positions of the SNP markers that mapped to the M432 LGs were consistent with their positions on the 'Golden Delicious' genome sequence and in some cases (LG3, LG6, LG7, LG11 and LG14) distinct domains signifying high and low recombination along the LG/pseudochromosome could be observed. However, each of the 17 LGs contained a small number of markers from more than one
'Golden Delicious' pseudo-chromosome, indicating the erroneous placement of genome sequence contigs in the 'Golden Delicious' pseudo-chromosomes or the presence of undetected gene paralogues within the Malus genome. A total of 311 Malus SNP markers mapped to positions on the M432 LGs that did not agree with their physical positions on the 'Golden Delicious' genome sequence. The markers were distributed throughout the 17 Malus pseudo-chromosomes and represent $13.7 \%$ of all markers mapped in the M432 mapping progeny. Marker genetic and physical positions are given in Additional file 1.

\section{Discussion}

We have extended the previously published M432 genetic linkage map using SNP markers generated using the IRSC array, and increased marker saturation on the map from an average of one marker every $3.8 \mathrm{cM}$ to one marker every $0.5 \mathrm{cM}$. Of the 7,867 Malus SNP markers contained on the array, 1,841 (23.4\%) were heterozygous in one of the two parents of the progeny, and a further

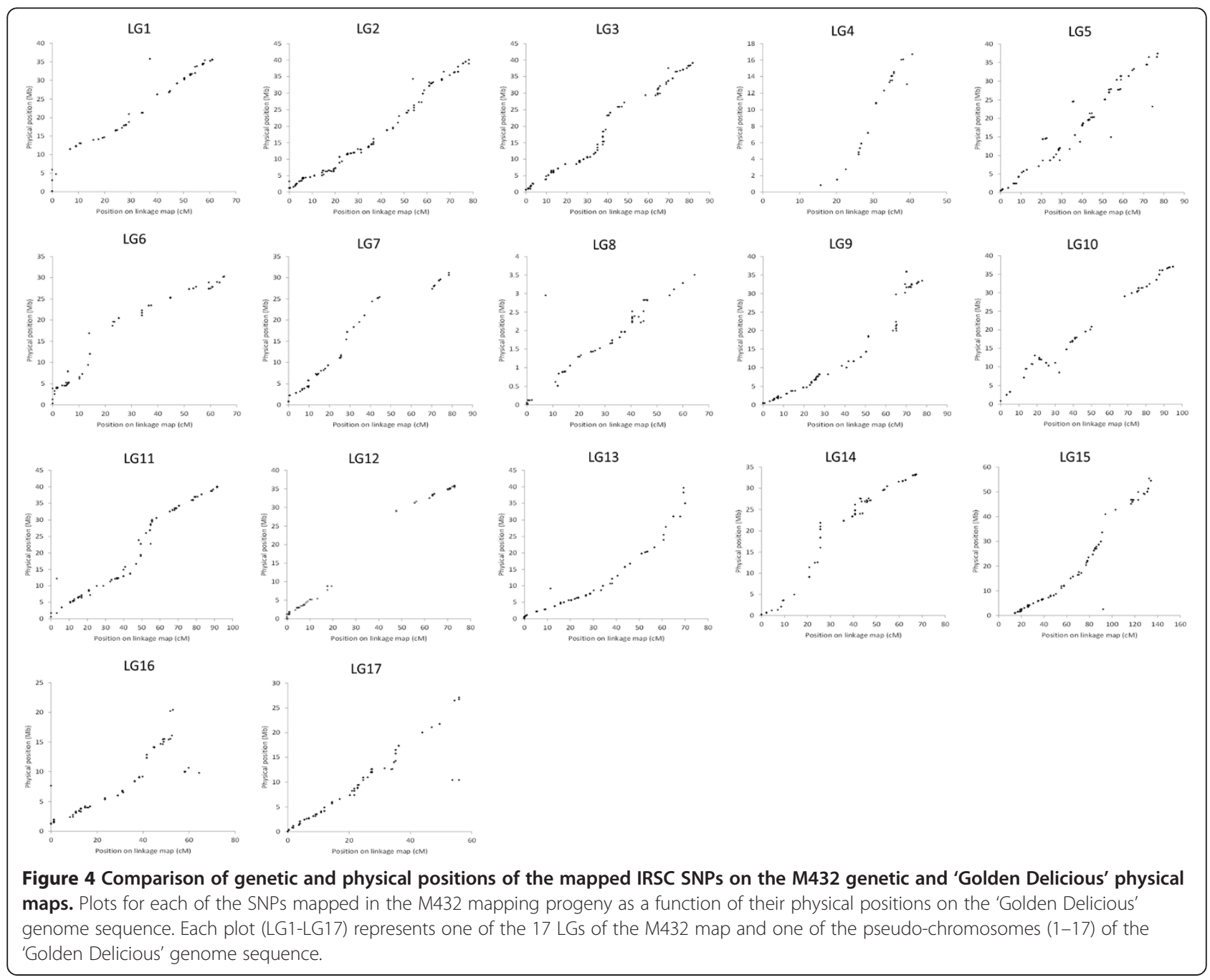


998 (12.6\%) were heterozygous in both parental genotypes. A total of 26 heterozygous SNPs were derived from the 921 Pyrus SNPs on the array, indicating a $\mathrm{T}_{\mathrm{SNP}}$ value of $2.82 \%$ transferability to Malus, which is within the range of values previously obtained by Micheletti et al. [5] of between 1.2 and 3\% transferability of SNPs from Malus to Pyrus. The total percentage of heterozygous Malus SNPs (36\%) was within the $\mathrm{T}_{\mathrm{SNP}}$ value range reported for the transferability of 'Golden Delicious' SNPs to rootstock varieties [5], indicating that previously reported $\mathrm{T}_{\mathrm{SNP}}$ values for Malus cultivars should be broadly applicable to the number of SNPs expected to segregate in a given Malus genotype on the IRSC array.

\section{SNP marker heterozygosity and GenomeStudio genotype clustering}

Our investigation revealed a large number of SNPs putatively heterozygous and thus segregating in the M432 progeny, but for which genotype calls using GenomeStudio (Illumina) resulted in segregation patterns that could not be assigned a position on one of the 17 LGs of the M432 consensus map. A closer inspection of the genotype data in GenomeStudio (Illumina) revealed low cluster separation calls for all SNPs and a number of different causes for the discrepancies in these data, including many loci for which data were grouped into more than one sub-cluster in each genotype. Sub-clusters are most likely caused by probes detecting more than one locus, one of which contained an SNP or indel, or the detection of more than one allele containing SNPs within the probe sequence [4], leading to non-uniform clustering of the detected genotypes. Such variation has been detected using oligonucleotide arrays in humans, where it is attributed to copy-number variation (CNV) [10]. Sanzol [11] investigated duplicated genes in Malus using EST data and concluded that the data supported a model of continuous small-scale duplication events, in addition to the recent whole genome duplication event in the lineage of the genus $[1,12]$. We suggest that the apparently large number of loci for which segregation data was generated using the IRSC array, but which could not be located to the M432 linkage map (20\%) is a result of a combination of factors, including detection of paralogous loci generated through whole genome duplication and $\mathrm{CNV}$, and the mis-assignation of genotypes scored in GenomeStudio (Illumina) due to the lack of a well defined cluster file for the analysis of Malus.

\section{SNP and SSR co-segregation analysis and linkage map construction}

To our knowledge, this is the most extensive linkage map of a Malus mapping progeny published to date. Previously, the IRSC array had been used to genotype the progeny derived from the cross 'Royal Gala' $\times$ ' Granny Smith' (RG $\times$ GS) which was reported by Chagné et al. [7], although in the report, data from only a single LG (LG1) was presented. The number of SNP markers heterozygous in the M432 population was 2,856 (32.5\% of the total number of SNPs on the array), almost identical to the 2,810 (32\% of the total number of SNPs on the array) reported to be heterozygous in $\mathrm{RG} \times \mathrm{GS}$ by Chagné et al. [7]. Of those markers heterozygous in the M432 progeny, 2,272 markers could be assigned reliable positions on the linkage map presented. Since only one LG was presented in the report of Chagné et al. [7], it was not possible to determine if all 2,810 markers were located to the RG $\times$ GS linkage map. Additionally as only markers segregating in a 'pseudo test-cross' configuration were presented on the $\mathrm{RG} \times \mathrm{GS}$ LG1 parental linkage maps and the M432 progeny is derived from a backcross between 'M.27' and its semi-vigourous seedling 'M.116' ('M.27’ × 'M.M.106'), direct comparison between the number of markers mapped in both investigations was not possible. However, the average distance between markers on the RG $\times$ GS linkage map was reported to be 0.88 and 0.91 for the 'Royal Gala' and 'Granny Smith' linkage maps respectively. These distances are comparable to the average genetic distances between the same marker types in this investigation (data not shown) and thus we conclude that, given the diverse pedigrees of these two mapping progenies, the IRSC array should be an invaluable tool for linkage map development and saturation in any given $M$. pumila mapping progeny.

We identified a number of regions of the M432 map where segregation distortion was widespread and extensive. The most extreme example was at the bottom of LG17 where the self-incompatibility is located [13,14]. The M432 progeny was derived from a semi-compatible cross between 'M.27' and its semi-vigourous seedling 'M.116' ('M.27' × M.M.106'), and thus fertilisation could not occur in homozygous genotype combinations around the $S$-locus, leading to the observed segregation distortion. Two other regions of distortion are probably the result of genes associated with the dwarfing phenotype in the cross, since some seedlings of the progeny displayed lethal and sub-lethal dwarf phenotypes. Efforts are on-going to characterise the M432 progeny and identify QTL associated with the dwarfing phenotype as well as a number of other traits including water-use efficiency.

\section{Genome heterozygosity, SNP marker genome coverage} and a comparison of genetic and physical SNP positions When the genetic positions of the 2,272 Malus SNP markers mapped in the M432 progeny were compared to their physical positions on the 'Golden Delicious' genome sequence a high degree of colinearity was observed. Additionally, distinct regions of high and low recombination could be observed in a number of cases along the 
LGs/pseudochromosomes. The anchored portion of the 'Golden Delicious' genome from which the physical positions of the SNPs mapped in the M432 progeny were derived covers an estimated $71.2 \%$ of the 'Golden Delicious' genome and represents the majority of the gene-rich portion of the apple genome sequence [1]. Since the un-sequenced and un-anchored portions of the genome have been predicted to be largely repetitive sequence, and the SNPs positioned on the IRSC array were derived almost exclusively from exonic sequence [7], it was expected that plots of genetic and physical positions of the SNPs mapped in the M432 progeny would reveal a largely linear relationship for each chromosome due to the absence of markers mapped to the largely repetitive centromeric and telomeric regions of the chromosomes. However, many of the plots revealed non-linear relationships, with clear regions of high recombination representing chromosome arms, and centres of low recombination representing putative centromeric regions, as have been observed in similar plots of other organisms such as Caenorhabditis elegans [15] and Oryza sativa [16]. Regions of effectively zero recombination at the LG ends which would indicate the presence of telomeric regions were largely undetected in this investigation. However, at least one region of zero recombination was observed on plots of LG/pseudochromosomes LG1, LG6, LG7 and LG12.

A total of 311 SNP markers (13.7\% of all mapped SNP markers) were located to positions on LGs that conflicted with the predicted positions of the SNPs on the 'Golden Delicious' pseudo-chromosomes [1]. Upon closer inspection of the predicted positions of these SNP markers, 47 (15\%) mapped to regions in the M432 mapping progeny that were homeologous to those predicted, whilst the remaining 264 (85\%) were located in non-homeologous regions of the genome, indicating the presence of paralogous genomic regions, or possible mis-assignment of genome sequence contigs during the assembly and anchoring of the 'Golden Delicious' genome sequence.

Human and animal genetics employ genetic information gained from individuals who are members of extended pedigrees for the identification of loci controlling complex genetic traits $[17,18]$. One of the aims of the IRSC was to produce a genotyping tool to enable the determination of SNP haplotypes for apple varieties related through well-defined pedigrees [7], leading to the identification of genes controlling important agronomic traits using a pedigree-based analysis. The advantages of such analyses are that the full variation associated with a trait of interest can be sampled within a gene pool; however, it is essential that markers included in each haplotype are derived from physically associated locations on the Malus genome. If the $13.7 \%$ of the markers mapped in the M432 progeny in this investigation that were identified as misplaced on the 'Golden Delicious' genome sequence of Velasco et al. [1], had been used to create haplotypes for pedigree-based association studies, they would have exhibited recombination at higher frequencies than linked markers within the haplotype, making genetic data within the pedigree difficult or impossible to interpret. In total, 2,272 (25.8\%) of the SNP markers contained on the IRSC array were mapped in the M432 progeny. If the markers mapped in the M432 progeny are representative of all SNPs contained on the array, there are potentially a further 767 SNPs for which the genetic and predicted genomic positions may disagree, potentially leading to the creation of false haplotypes for Malus cultivars.

\section{Conclusions}

We have employed the IRSC Malus array to extend and saturate the SSR linkage map of the M432 mapping progeny. The use of the array enabled us to locate a total of 2,270 SNPs to the consensus linkage map at a fraction of the time and cost of developing a similar map using other experimental approaches, and to develop the most comprehensive saturated complete linkage map for a Malus mapping progeny to date. The mapping of the SNPs enabled us to assess the relative coverage and accuracy of the 'Golden Delicious' genome sequence and to identify a significant proportion (13.7\%) of SNPs that had been erroneously located to the 'Golden Delicious' pseudochromosomes. Further mapping in additional progenies will help to characterise regions of the 'Golden Delicious' sequence that are incorrectly placed in the current assembly version, and assist in the continued improvement of the reference sequence for Malus.

\section{Methods}

\section{Plant material and DNA extraction}

The M432 rootstock progeny used in this investigation was first described by Evans et al. [8]. It was derived from the cross 'M.27' × 'M.116' ('M.M.106' × M.27') and comprises 140 seedlings. DNA from the seedlings of the M432 progeny was used to generate SNP data using the IRSC array, and subsequently to construct SNP-based linkage maps of the parental genomes. DNA was freshly isolated from young leaf tissue of 'M.27, 'M.116' and the 140 seedlings of the M432 progeny using the DNeasy plant miniprep kit (Qiagen) according to the manufacturer's protocol immediately prior to genotyping. It was then quantified using PicoGreen (Invitrogen) against a $\lambda$ standard DNA dilution series using a Synergy 2 fluorimeter (BioTek). DNA from all seedlings was diluted to $50 \mathrm{ng} / \mathrm{ul}$ for genotyping using the IRSC array.

\section{Malus Infinium ${ }^{\circledR}$ II whole genome genotyping array}

The IRSC array, employing exclusively Illumina Infinium ${ }^{\circledR}$ II design probes and dual colour channel assays (Infinium HD Assay Ultra, Illumina), that was described previously 
by Chagné et al. [7] was used for genotyping the progeny of the M432 apple mapping population. The IRSC bead chips contained a total of 7,867 possible Malus, and 921 possible Pyrus SNPs. Progeny DNA was assayed following the manufacturer's recommendations. Briefly, a whole genome amplification reaction was performed, followed by denaturation and hybridization to the IRSC BeadChips (Illumina). Un-hybridized and non-specifically hybridized DNA was removed through washing, following which a single base extension reaction was performed to incorporate differentially labeled nucleotides at the SNP sites of each of the 8,788 probes of the BeadChip for each M432 genotype. BeadChips were then imaged and data were collected using the HiScan detection platform (Illumina) following Illumina-published standard operating procedures (http://www.illumina.com).

\section{Data scoring and SNP nomenclature}

Data generated for the 8,788 SNPs in the 140 seedlings and the two parents of the M432 progeny were scored using GenomeStudio (Illumina) using a GenCall threshold of 0.15. Data were then exported into Excel (Microsoft Inc.) for post scoring processing before data analysis. Prior to linkage analysis markers with a GenTrain score below 0.4 were excluded from the dataset. Following initial linkage analyses, all markers not mapping to one of the expected 17 LGs were visually inspected for accuracy, to determine data quality, and to resolve any errors created by automatic allele calling in GenomeStudio (Illumina). Data for all SNPs showing putative segregation were discarded prior to mapping if one or both of the parental genotypes failed to amplify in the assay or if segregation data contained genotypes not expected from the parental genotypes.

\section{Data analysis and M432 linkage map construction}

Markers were re-coded using genotype codes for linkage analysis using JO INMAP 4.0 (Kyasma, Wageningen, NL), according to their segregation type; $\mathrm{AB} \times \mathrm{AA}$ or $\mathrm{AB} \times \mathrm{BB}$ (segregating in the female 'M.27' genotype) were coded $1 \mathrm{~m} \times 11$ and $\mathrm{AA} \times \mathrm{AB}$ or $\mathrm{BB} \times \mathrm{AB}$ (segregating in the male 'M.116' genotype) were coded $n n \times n p$. Markers segregating $\mathrm{AB} \times \mathrm{AB}$ (in both parental genotyes) were recoded $h \mathrm{k} \times \mathrm{hk}$. SNP nomenclature in this investigation followed the dbSNP (EMBL) accession codes for each SNP on the array that has been deposited in the Genome Database for Rosaceae [19].

Linkage mapping was performed with all SNP data and the SSR data of Fernández-Fernández et al. [9]. Data for SSR markers with parental genotypes $A \varnothing \times A \varnothing$ (and thus segregating in a 3:1 Mendelian ratio) were not considered in this analysis. Segregation data for SNPs were analysed and consensus genetic linkage maps were obtained using a two-step procedure. Initially SNP markers were ordered using the QMAP procedure of Genstat 14 [20]. For outbreeders QMAP employs the method of Jansen [21] for ordering markers, in combination with the EM algorithm using a hidden Markov model for obtaining multipoint maximum likelihood estimates of recombination frequencies [22]. SNP marker orders were then fixed and SSRs were added to the consensus SNP linkage map using JOINMAP 4.0 (Kyazma, NL); marker placement was determined using a minimum LOD score threshold of 3.0, a recombination fraction threshold of 0.35 , ripple value of 1.0, jump threshold of 3.0 and a triplet threshold of 5.0, and mapping distances were calculated using the Kosambi mapping function. Linked markers were only considered as constituting a LG if more than six markers coalesced into a single group. Any markers that remained 'unlinked' following co-segregation analysis or that were contained in groups of fewer than six markers were visually inspected in GenomeStudio (Illumina). All linkage maps presented were plotted using MAPCHART 2.2 for Windows [23] and LG nomenclature for M432 follows the numbering reported previously for this progeny by Fernández-Fernández et al. [9].

\section{Comparison of genetic locations and physical positions on the 'Golden Delicious' genome sequence}

Physical positions of all SNP markers were derived from their predicted positions on the 'Golden Delicious' genome sequence and were plotted as a function of genetic distances on the M432 consensus map. Mareymaps for each individual chromosome were produced using Excel (Microsoft Inc.).

\section{Additional file}

Additional file 1: Segregation data for the 2,579 markers segregating in the M432 progeny. The 2,272 segregating IRSC array SNP markers

mapped in the M432 population along with the 307 previously mapped SSR and S-locus markers, including the SNP name, the dbSNP (EMBL) accession code assigned to each on the IRSC array, the LG and map position of each mapped marker, the monogenic marker segregation ratios, associated chi-squared values and the predicted pseudo-chromosome positions of each marker on the 'Golden Delicious' genome sequence. Markers for which genetic and physical positions conflicted are highlighted in red.

\section{Competing interests}

The authors declare that they have no competing financial interests.

\section{Acknowledgements}

Malus genetics and genomics are sponsored at East Malling Research by the Department for Environment Food and Rural Affairs. Malus genetics and genomics are funded at FEM-IASMA by the Autonomous Province of Trento (Italy). J. Jansen acknowledges support by the USDA National Institute of Food and Agriculture-Speciality Crop Research Initiative RosBREED (200951181-05808) and the FruitBreedomics project funded by the Commission of the European Communities (Contract FP7-KBBE-2010-265582).

\section{Author details}

${ }^{1}$ East Malling Research (EMR), New Road, East Malling, Kent ME19 6BJ, UK. ${ }^{2}$ Biometris, Wageningen University and Research Centre, P.O. Box 100, 6700 AC Wageningen, the Netherlands. ${ }^{3}$ Istituto Agrario San Michele all'Adige, Via E. Mach 1, 38010 San Michele all'Adige, Italy. ${ }^{4}$ WSU Tree Fruit 
Research \& Extension Center, 1100 N. Western Ave., Wenatchee, WA 988011230 USA. ${ }^{5}$ School of Biological Sciences, University of Reading, Reading RG6 $6 \mathrm{AH}, \mathrm{UK}$

\section{Authors' contributions}

LA carried out the experiments, analysed the data and co-authored the manuscript. FFF conceived the experiments, managed the populations and co-authored the manuscript. JJ analysed the data. EB carried out the experiments. KME conceived the experiments and critically evaluated the manuscript. RVio advised on the experiments. RVel managed the experiments. JMD advised on the experiments and critically evaluated the manuscript. MT conceived the experiments, managed the experiments, analysed the data and co-authored the manuscript. DJS conceived the experiments, managed the experiments, analysed the data and authored the manuscript. All authors read and approved the final manuscript

Received: 9 January 2012 Accepted: 25 May 2012

Published: 25 May 2012

\section{References}

1. Velasco R, Zharkikh A, Affourtit J, Dhingra A, Cestaro A, Kalyanaraman A Fontana P, Bhatnagar SK, Troggio M, Pruss D, et al: The genome of the domesticated apple (Malus x domestica Borkh.). Nat Genet 2010, 42:833-839.

2. Zharkikh A, Troggio M, Pruss D, Cestaro A, Eldrdge G, Pindo M, Mitchell JT, Vezzulli S, Bhatnagar S, Fontana P, et al: Sequencing and assembly of highly heterozygous genome of Vitis vinifera L. cv Pinot Noir: Problems and solutions. J Biotechnol 2008, 136:38-43.

3. Huo NX, Garvin DF, You FM, McMahon S, Luo MC, Gu YQ, Lazo GR, Vogel JP. Comparison of a high-density genetic linkage map to genome features in the model grass Brachypodium distachyon. Theor Appl Genet 2011, 123:455-464.

4. Pindo M, Vezzulli S, Coppola G, Cartwright DA, Zharkikh A, Velasco R, Troggio M: SNP high-throughput screening in grapevine using the SNPlex (TM) genotyping system. BMC Plant Biol 2008, 8:12.

5. Micheletti D, Troggio M, Zharkikh A, Costa F, Malnoy M, Velasco R, Salvi S: Genetic diversity of the genus Malus and implications for linkage mapping with SNPs. Tree Genet Genomes 2011, 7:857-868.

6. lezzoni A, Weebadde C, Luby J, Yue CY, Weg Evd, Fazio G, Main D, Peace CP, Bassil NV, McFerson J: RosBREED: Enabling marker-assisted breeding in Rosaceae. In Acta Horticulturae. Edited by Bassil NV, Martin R. 2010:389-394.

7. Chagné D, Crowhurst R, Troggio M, Davey MW, Gilmore B, Lawley C, Vanderzande S, Hellens RP, Kumar S, Cestaro A, Velasco R, Main D, Rees DJG, lezzoni A, Mockler T, Wilhelm L, Van de Weg E, Gardiner SE, Bassil N, Peace $\mathrm{C}$ : Genome-wide SNP detection, validation, and development of an $8 \mathrm{~K}$ SNP array for apple. PLoS One 2012, 7:e31745.

8. Evans KM, Fernandez-Fernandez F, Govan CL, Clarke JB, Tobutt KR: Development of a new apple rootstock framework map. In Acto Horticulturae. Edited by Robinson TL.: ; 2011:69-74.

9. Fernández-Fernández F, Antanaviciute L, van Dyk MM, Tobutt KR, Evans KM, Rees DJG, Dunwell JM, Sargent DJ: A genetic linkage map of an apple rootstock progeny anchored to the Malus genome sequence. Tree Genet Genomes (online first) 2012, doi:10.1007/s11295-012-0478-7.

10. Franke L, de Kovel CGE, Aulchenko YS, Trynka G, Zhernakova A, Hunt KA, Blauw $H$ M, van den Berg LH, Ophoff R, Deloukas P, van Heel DA, Wijmenga C: Detection, imputation, and association analysis of small deletions and null alleles on oligonucleotide arrays. Am J Hum Genet 2008, 82:1316-1333.

11. Sanzol J: Dating and functional characterization of duplicated genes in the apple (Malus domestica Borkh.) by analyzing EST data. BMC Plant Biol 2010, 10:87.

12. Jung $S$, Cestaro A, Troggio M, Main D, Zheng P, Cho I, Folta KM, Sosinski B, Abbott AG, Celton JM, Arús P, Shulaev V, Verde I, Morgante M, Rokhsar DS, Velasco R, Sargent DJ: Whole genome comparisons of Fragaria, Prunus and Malus reveal different modes of evolution between Rosaceous subfamilies. BMC Genomics 2012, 13:129.

13. Bošković R, Tobutt KR: Correlation of stylar ribonuclease isoenzymes with incompatibility alleles in apple. Euphytica 1999, 107:29-43.

14. Maliepaard C, Alston FH, van Arkel G, Brown LM, Chevreau E, Dunemann F Evans KM, Gardiner S, Guilford P, van Heusden AW, et al: Aligning male and female linkage maps of apple (Malus pumila Mill.) using multi-allelic markers. Theor Appl Genet 1998, 97:60-73.

15. Rockman MV, Kruglyak L: Recombinational landscape and population genomics of Caenorhabditis elegans. PLoS Genet 2009, 5:e1000419.
16. Tian ZX, Rizzon C, Du JC, Zhu LC, Bennetzen JL, Jackson SA, Gaut BS, Ma JX: Do genetic recombination and gene density shape the pattern of DNA elimination in rice long terminal repeat retrotransposons? Genome Res 2009, 19:2221-2230.

17. Barendse W, Armitage SM, Kossarek LM, Shalom A, Kirkpatrick BW, Ryan AM, Clayton D, Li L, Neibergs HL, Zhang N, et al: A genetic-linkage map of the bovine genome. Nat Genet 1994, 6:227-235.

18. Wegmann D, Kessner DE, Veeramah KR, Mathias RA, Nicolae DL, Yanek LR, Sun YV, Torgerson DG, Rafaels N, Mosley T, et al: Recombination rates in admixed individuals identified by ancestry-based inference. Nat Genet 2011, 43:847-853.

19. Jung $S$, Staton M, Lee T, Blenda A, Svancara R, Abbott A, Main D: GDR (Genome Database for Rosaceae): integrated web-database for Rosaceae genomics and genetics data. Nucleic Acids Res 2008, 36(Database issue): D1034-D1040.

20. Payne RW, Harding SA, Murray DA, Soutar DM, Baird DB, Glaser Al, Welham SJ, Gilmour AR, Thompson R, Webster R: GenStat ${ }^{\circledR}$ Release 14 Reference Manual. Hemel Hempstead, UK: VSN International; 2012.

21. Jansen J: Construction of linkage maps in full-sib families of diploid outbreeding species by minimizing the number of recombinations in hidden inheritance vectors. Genetics 2005, 170:2013-2025.

22. Lander ES, Green P: Construction of multilocus genetic linkage maps in humans. Proc Natl Acad Sci U S A 1987, 84:2363-2367.

23. Voorrips RE: MapChart: Software for the graphical presentation of linkage maps and QTLs. J Hered 2002, 93:77-78.

\section{doi:10.1186/1471-2164-13-203}

Cite this article as: Antanaviciute et al:: Development of a dense SNPbased linkage map of an apple rootstock progeny using the Malus Infinium whole genome genotyping array. BMC Genomics 2012 13:203.

\section{Submit your next manuscript to BioMed Central and take full advantage of:}

- Convenient online submission

- Thorough peer review

- No space constraints or color figure charges

- Immediate publication on acceptance

- Inclusion in PubMed, CAS, Scopus and Google Scholar

- Research which is freely available for redistribution

Submit your manuscript at www.biomedcentral.com/submit
C BioMed Central 